\title{
INFLUÊNCIA DA GEOMETRIA DO CORPO DE PROVA NA OBTENÇÃO DA CLC 0 DO LATÃO 90/10 *
}

\section{Resumo}

Leandro de Almeida ${ }^{1}$ Gustavo Tressia ${ }^{2}$

Victor Caso Moreira ${ }^{3}$

As curvas limite de conformação (CLC) são utilizadas há mais de 50 anos. Propostas inicialmente para 0 desenvolvimento de produtos provenientes da indústria automobilística, em função das necessidades apresentadas pela indústria de estampagem elas são aplicadas atualmente de maneira geral na produção de inúmeros tipos de peças, oferecendo previsões de possíveis falhas e/ou defeitos durante as etapas do processo de estampagem. As CLC's permitem prever o afinamento excessivo, enrugamento, estiramento e é claro, a fratura da peça. A raiz da curva limite de conformação (CLC $)$ é considerada como a mínima deformação obtida pela curva, ou seja, a obtenção de deformações superiores pode levar a peça a falha. As ligas de latão compostas por cobre e zinco, são utilizadas em inúmeras aplicações que necessitam de excelente estampabilidade, como o repuxo profundo, objetivado na fabricação de projéteis. Desta forma, torna-se interessante o entendimento da CLC ou CLCo para liga de latão em estudo. A obtenção da CLC $_{0}$ através de ensaios de tração uniaxial no plano da chapa pode ser realizada de acordo com corpos de prova de formatos específicos, como os propostos por Björklund e Nilsson (2014), IRSID (1975) e Wagoner (1980 e 1981). Concluiu-se que o corpo de prova proposto pelo IRSID (1975) é o que melhor se apresentou na determinação das deformações $\varepsilon_{1} \times \varepsilon_{2}$ e com rompimentos dos corpos de prova na região central.

Palavras-chave: Latão; Curva limite de conformação; ensaio de tração uniaxial; estampagem.

\section{INFLUENCE OF SAMPLE GEOMETRY ON THE FLC 0 MEASUREMENT FOR THE 90/10 BRASS}

\begin{abstract}
The forming limit curves (FLC) have been used for more than 50 years. Initially, they were proposed for the development of products from the automobile industry. As the drawing industry advanced, the FLC are now applied in general to the production of numerous types of parts, offering predictions of possible failures and / or defects during the stamping process steps. The FLCs allow to predict excessive thinning, wrinkling, stretching and of course, the fracture of the part. The root of the forming limit curve $\left(F L C_{0}\right)$ is considered the minimum deformation of the curve, that is, higher deformations can cause the part to fail. The brass alloys composed of copper and zinc are used in numerous applications that need excellent stamping, such as the deep drawing, aimed at the manufacture of projectiles. In this way, the understanding of FLC or $\mathrm{FLC}_{0}$ for the studied brass alloy becomes interesting. In order to obtain the $\mathrm{FLC}_{0}$ by uniaxial tensile tests in the plane of the plate, it was used specimens of specific shapes, such as those proposed by Björklund and Nilsson (2014), IRSID (1975) and Wagoner (1980 and 1981). It was concluded that IRSID (1975) was the best geometry for the determination of the deformations $\varepsilon_{1} \times \varepsilon_{2}$, add to the fact that the rupture of the specimen was in the central region.
\end{abstract}

Keywords: Brass; Forming limit curve; uniaxial tensile tests; formability.

1 Engenheiro Metalurgista, Engenheiro de Processos, Paranapanema SA, Santo André, SP, Brasil. 2 Engenheiro Mecânico, Mestre, Doutorando da Universidade de São Paulo, São Paulo, SP, Brasil.

3 Engenheiro Metalurgista, Mestre, Engenheiro de Processos, Paranapanema SA, Santo André, SP, Brasil. 


\section{INTRODUÇÃO}

A curva limite de conformação (CLC) é utilizada como uma ferramenta de grande valia na indústria de estampagem e principalmente para fabricação de peças na indústria automobilística. Seu objetivo principal é verificar o lugar correspondente geometricamente dos máximos pontos de deformação de uma determinada chapa metálica submetida a esforços, representando de maneira ampla a estampabilidade de um determinado material.

O lado direito da curva limite de conformação $\left(\varepsilon_{2}>0\right)$, foi descoberto em 1963 em um trabalho publicado por Keller e Backofen [1], em houve a descoberta do surgimento da estriçcão, associado a valores críticos das duas deformações principais no plano da chapa, através de um ensaio de estiramento. O lado esquerdo $\left(\varepsilon_{2}<0\right)$ foi determinado por Goodwin somente em 1968. Desde então, a curva limite de conformação é chamada de diagrama de Keller-Goodwin e vem sofrendo inúmeras evoluções com relação à forma de obtenção dos dados (ensaios).

O ponto mais importante da curva limite de conformação é conhecido como CLCo e é denominado como a raiz da curva limite de conformação, sendo posicionado no caminho da deformação planar no ponto de menor deformação $\varepsilon_{1}$ e a deformação $\varepsilon_{2}$ $=0$. Qualquer deformação alcançada abaixo deste valor no processo de estampagem garante que a peça fabricada não sofrerá um colapso, seja ele em qualquer caminho de deformação e principalmente na deformação planar. Ayres, Brazier e Sajewski [2] descrevem que de 80 a $85 \%$ das falhas que ocorrem na fabricação de peças produzidas pela indústria automobilística são pertinentes ao caminho de deformação planar e consecutivamente ao posicionamento da $\mathrm{CLC}_{0}$, com a menor deformação $\left(\varepsilon_{2}\right)$ variando entre $\pm 2 \%$, o que caracteriza a deformação planar.

As curvas limites de conformação são obtidas de inúmeras formas, podendo ser realizados ensaios como Nakazima, Marciniak e ensaios de tração uniaxial com corpos de prova de diferentes geometrias, como os propostos por Holmberg, Enquist e Thilderkvist [3], e/ou os propostos pelo IRSID ("Institut de Recherches de la Sidérurgie"), desenvolvido pelo laboratório central de pesquisas do grupo siderúrgico Frances Usinor em 1975. As metodologias apresentam diferenças significativas que devem ser levadas em consideração com relação aos resultados obtidos. No ensaio Nakazima utiliza-se um punção de formato hemisfério e no ensaio de Marciniak utilizase um punção de formato plano, sendo assim os resultados obtidos são diferentes. Os ensaios realizados no plano da chapa, ou seja, nos ensaios de tração uniaxial a deformação obtida é inferior em função da forma que os corpos de prova foram ensaiados.

A Figura 1 demonstra uma diferença significativa nos processos de obtenção das curvas limites de conformação apenas no lado esquerdo, em função dos corpos de prova de ensaio de tração uniaxial utilizados. A geometria dos corpos de prova é fundamental para se obter grandes deformações; os raios de curvatura dos entalhes dos corpos de prova devem aliviar a maior concentração de tensão possível, desta forma possibilitando maior deformação e se aproximando dos resultados obtidos por ensaios realizados com punções [3]. 


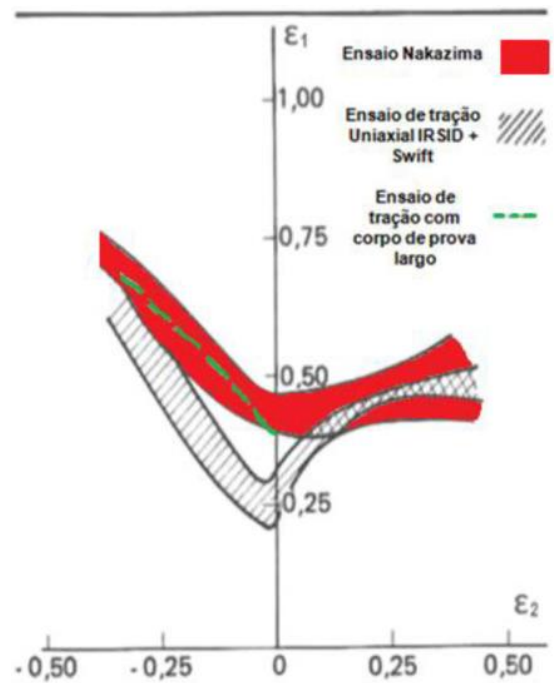

Figura 1 Comparativo da curva limite de conformação obtida pelo método IRSID x Nakazima e tração com corpo de prova de largo (USIMINAS, [4]).

O objetivo do presente trabalho é avaliar o ponto mais importante da curva limite de conformação, a curva raiz CLC $_{0}$ da liga de latão 90/10 (UNS C22000), utilizada principalmente na fabricação de projetil e que necessita de um repuxo profundo em função da aplicação, portanto, a obtenção da deformação planar realizada através de ensaios de tração uniaxiais, facilitará a obtenção da deformação e ajudará na prevenção de possíveis ocorrências quando da utilização do material.

Wagoner [5] publicou um artigo sobre a análise e medição da deformação planar em alumínio 2036 - T4 com corpos de prova possuindo diferentes geometrias. Um dos seus objetivos foi avaliar a influência do ângulo, raio e abertura horizontal do entalhe nos corpos de prova na concentração de tensão e consecutivamente sua influência na obtenção da deformação planar, utilizando-se também a análise por elementos finitos. Seus resultados demonstram que a maior deformação é obtida para a condição H ilustrada na Figura 2.

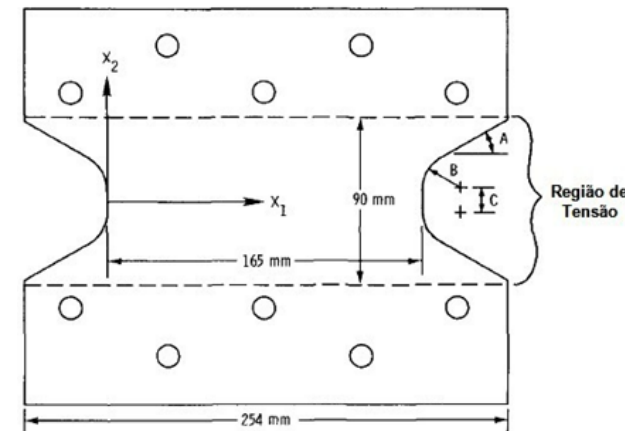

\begin{tabular}{c} 
Corpos de prova \\
\hline$A$ \\
$B$ \\
$C$ \\
$D$ \\
$E$ \\
$F$ \\
$G$ \\
$H^{*}$
\end{tabular}

\begin{tabular}{c}
$A$, deg \\
\hline 0 \\
45 \\
30 \\
45 \\
30 \\
45 \\
0 \\
-
\end{tabular}

\begin{tabular}{cr}
$B, \mathrm{~mm}$ & $C, \mathrm{~mm}$ \\
\hline 19.1 & 12.7 \\
19.1 & 12.7 \\
12.7 & 12.7 \\
12.7 & 12.7 \\
19.1 & 12.7 \\
25.4 & 0.0 \\
12.7 & 12.7 \\
68.4 & 0.0
\end{tabular}

Figura 2 Corpo de prova para obtenção da deformação planar e variações dimensionais de A a

$\mathrm{H}$ para as cotas A, B e C de diferentes corpos de provas (adaptado de [5]).

Björklund e Nilsson [6] estudaram a deformação planar no aço dual-phase utilizando corpos de prova de geometrias bem inferiores às geometrias apresentadas por Wagoner. O objetivo do estudo foi verificar os tipos de falhas ocorridas em corpos de prova de deformação planar e de ensaio Nakazima, concluindo-se que para o caminho de deformação planar, ocorre certa instabilidade na deformação em decorrência do aumento da velocidade antes da ocorrência da fratura do corpo de 
prova, estando esse aumento de velocidade diretamente relacionado ao tipo de material ensaiado e sua estrutura.

Xavier e Plaut [7] avaliaram a determinação da CLC 0 através da deformação planar obtida em corpos de prova similares aos apresentados por Wagoner, esses resultados foram comparados com os obtidos em ensaio Nakazima para os aços IF (livre de intersticiais) e o aço 1050 coalescido, concluindo que o ensaio realizado no plano não sofre a influência da geometria do punção e consecutivamente do atrito entre o punção e o material a ser ensaiado, desta forma apresenta um valor de deformação planar inferior ao apresentado pelo ensaio Nakazima.

Schwindt et al [8] estudaram para o aço DP - 780 o levantamento da curva limite de conformação através da metodologia proposta pelo IRSID (ensaio de tração uniaxial) e o ensaio de Nakazima reduzido em $60 \%$. A principal conclusão é que na deformação planar existe uma diferença de $70 \%$ entre as metodologias, pois ensaios realizados no plano, como os de tração uniaxial, não sofrem a influência da geometria do punção e tampouco do atrito entre o material e o punção. Sendo assim, a deformação apresentada pelo ensaio Nakazima é superior.

\section{MATERIAIS E MÉTODOS}

A composição química do material estudado é apresentada na Tabela 1 e foi obtida por meio de análise em fluorescência de raios-X.

Tabela 1 Composição química.

\begin{tabular}{ccccccc}
\hline \multirow{2}{*}{ Amostra } & \multicolumn{7}{c}{ Composição química, em \% mássica } \\
\cline { 2 - 7 } & $\mathbf{C u}$ & $\mathbf{F e}$ & $\mathbf{S n}$ & $\mathbf{C r}$ & $\mathbf{C d}$ & Zn \\
\hline & 89,7700 & 0,0027 & 0,0045 & 0,0071 & 0,0019 & 10,2138 \\
\hline
\end{tabular}

As amostras foram produzidas incialmente pelo processo chama semicontínuo, de fusão do metal líquido e vazamento em molde metálico, obtendo-se uma placa que posteriormente foi aquecida e laminada a quente com redução de espessura em torno de $90 \%$. A laminação a frio foi realizada através da redução de espessura de $85 \%$, seguida do recozimento a $540^{\circ} \mathrm{C}$ por um período de 720 minutos obtendo-se um tamanho de grão médio de $30 \mu \mathrm{m}$, sendo o tamanho de grão, determinado pelo método comparativo da ASTM E112 [9]. A laminação a frio de acabamento foi realizada com redução de espessura de $40 \%$ seguida do recozimento a $450^{\circ} \mathrm{C}$ por um período de 720 minutos com a obtenção de um tamanho de grão médio de $15 \mu \mathrm{m}$, determinado da mesma forma que a do material intermediário (ASTM E112).

A curva limite de conformação raiz $\left(C L C_{0}\right)$ é o ponto mais importante da CLC, conforme descrito anteriormente. Em função de sua importância, julgou-se necessário comparar os diferentes métodos desenvolvidos por Wagoner [5, 10], Björklund e Nilsson [6] e proposto pelo IRSID [11].

Para determinação da deformação planar foram confeccionados três corpos de prova de cada autor, ou seja, três corpos de prova do Björklund e Nilsson [6], Figura 3 , três corpos de prova com dimensões seis vezes menor ao proposto por Wagoner [10], com comprimento de $150 \mathrm{~mm}$, corpo de prova tipo $\mathrm{H}$, vide Figura 3 e três corpos de prova igual ao desenvolvido pelo IRSID vide Figura 3. 


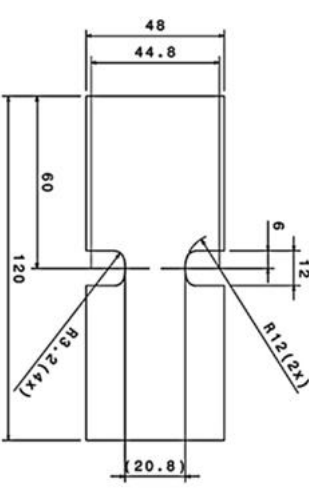

Björklund e Nilsson

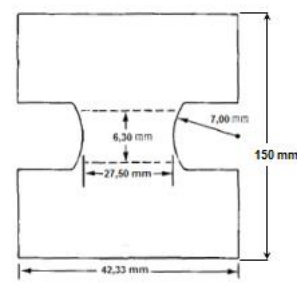

Wagoner

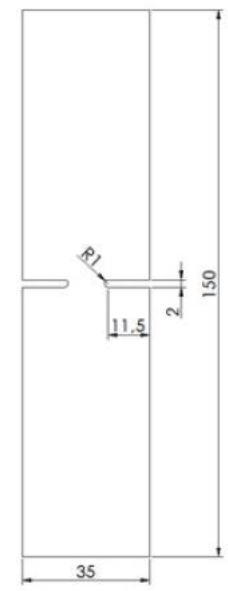

IRSID

Figura 3 Corpo de prova para obtenção da deformação planar (adaptado de $[6,10,11]$ ).

Os corpos de prova foram elaborados na eletroerosão em função da precisão dimensional e do excelente acabamento obtido pelo processo. As gravações dos círculos para medição da deformação planar foram efetuadas pelo processo de corrosão eletroquímica nas regiões centrais dos corpos de prova. As circunferências foram medidas antes e após os ensaios de tração uniaxial no microscópio estéreo Olympus, modelo SZX7 Zoom. Os ensaios de tração foram efetuados na máquina de tração Tinus Olsen, modelo H25 KL, com velocidade fixa de $15 \mathrm{~mm} / \mathrm{min}$.

\section{RESULTADOS E DISCUSSÃO}

Os resultados de curva limite de conformação raiz $\left(C L C_{0}\right)$ para os três corpos de prova estudados são apresentados na Figura 4. Observa-se que os três ensaios não são coincidentes, ou seja, não se sobrepõem no gráfico $\varepsilon_{1} \times \varepsilon_{2}$. O método proposto por IRSID [11] apresenta-se mais próximo da condição $\varepsilon_{2}=0$, e menor valor de $\varepsilon_{1}$. De maneira oposta, o método proposto Björklund e Nilsson [6] apresenta-se mais distante da condição de deformação planar, e maior valor de deformação $\varepsilon 1$.

Ayres, Brazier e Sajewski [2] afirmam que 80 a $85 \%$ das falhas que ocorrem na deformação plana (CLCo) durante a fabricação de peças automotivas, possuem a deformação menor $\varepsilon_{2}$ variando de $-0,02<\varepsilon_{2}>0,02$. Desta forma, a metodologia que mais se aproximou desta condição foi a proposta pelo IRSID, visto que os resultados detectados nos outros corpos de prova, atingiram valores próximos a 0,062.

Os corpos de prova possuem raios de curvaturas dos entalhes distintos. O corpo de prova que apresentou maior deformação $\varepsilon_{1}$, possui um raio de $12 \mathrm{~mm}$, em contrapartida o corpo de prova proposto pelo IRSID possui o raio do entalhe de $1 \mathrm{~mm}$. Em um estudo comparativo entre testes físicos e simulação computacional, Holmberg, Enquist e Thilderkvist [3] avaliaram a importância do raio, largura e comprimento do entalhe dos corpos de prova para levantamento da curva limite de conformação, efetuada através de ensaio de tração uniaxial, concluindo que os raios de curvatura devem aliviar a maior concentração de tensão possível. Portanto, quanto maiores os raios, menor será a concentração de tensão obtida, o que foi confirmado nos corpos de prova propostos por Björklund e Nilsson [6]. A largura influencia significativamente o estado de tensão e os corpos de prova devem possuir a largura o maior possível, já 
o comprimento é o inverso, quanto menor melhor, pois se obtém uma condição mais próxima da deformação plana na região central dos corpos de prova.

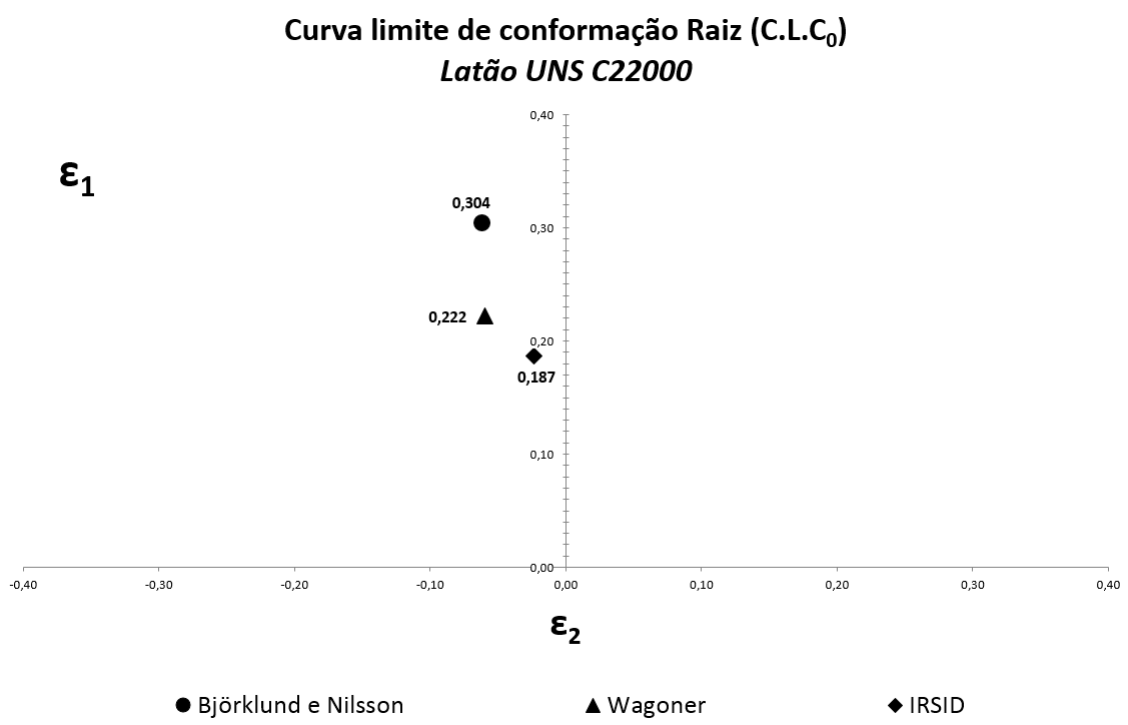

Figura 4 Curva limite de conformação raiz $\left(\mathrm{CLC}_{0}\right)$.

A altura do entalhe tem uma grande influência sobre a distribuição de tensões e deformações nos corpos de prova. Com uma altura do entalhe muito grande, a deformação nas bordas se tornará grande e a fratura ocorrerá nas bordas dos corpos de prova e não na região central desejada.

A diferença de deformação apresentada está diretamente relacionada com os raios de confecção dos entalhes dos corpos de prova, pois corpos de prova com raios mais acentuados concentram uma quantidade de tensão superior e diminuem a deformação que o material alcança. A largura e comprimento dos entalhes também influenciam principalmente na deformação $\varepsilon_{1}$, desta forma um balanceamento destas variáveis deve ser levado em consideração para se obter a melhor deformação planar do material.

O corpo de prova que mais caracteriza a curva limite de conformação raiz é o tipo proposto pelo IRSID [11], devido a baixa deformação $\varepsilon 2$ encontrada e o rompimento centralizado durante os ensaios.

Conforme o trabalho de Schwindt et al [8] sugere, deve-se ter em mente que a deformação obtida por este ensaio deve ser cerca de $60 \%$ a $70 \%$ inferior à deformação que seria obtida por ensaio de punção, como o Nakazima, por conta da ausência de atrito.

\section{CONCLUSÃO}

O corpo de prova que mais se aproximou da deformação planar (CLC $)$ foi o proposto pelo IRSID, em função da menor deformação ع2 obtida. As geometrias dos corpos de prova possuem grande influência na deformação final obtida, principalmente o raio dos entalhes. 


\section{Agradecimentos}

Os autores agradecem a Paranapanema SA por apoiar este e outros trabalhos.

\section{REFERÊNCIAS}

1 KELLER, P.S. e BACKOFEN, W.A. Plastic instability and fractury in sheets stretched over rigid punches. Metallurgical Transactions, Volume 56, 1963, 25-48 p.

2 AYRES, A.R.; BRAZIER, G.W.; SAJEWSKI, F.V. Evaluating the GMR-Limiting dome height test as a new measure of press formability near plane strain. J. Applied Metal Working, Volume 1, American Society for metal, 1979, 41 - 49 p.

3 HOLMBERG, S.; ENQUIST, B.; THILDERKVIST P. Evaluation of sheet metal formability by tensile tests, Journal of Materials Processing Technology 145, 2004, $72-83 \mathrm{p}$.

4 USIMINAS, Conformação na prensa - fascísculo de informações técnicas. №6, 1999, 58 pp.

5 WAGONER, R.H. Measurement and analysis of plane-strain work hardening. Metallurgical transactions, Volume 11A, January, 1980. 165 - 175 p.

6 BJÖRKLUND, O.; NILSSON, L. Failure characteristics of a dual-phase steel sheet, Journal of Materials Processing Technology, 2014. $1190-1204$ p.

7 PLAUT, R.L. Estampabilidade de chapas: ensaios e aplicações. In: Textura e relações de orientação: deformação plástica, recristalização, crescimento de grão. São Paulo: IPEN, 2003. 265 - 308 p.

8 SCHWINDT, C. D.; STOUT, M.; IURMAN, L.; SIGNORELLI, J. W. Forming limit curve determination of a DP-780 steel sheet. International congress of science and technology of metallurgy and materials, SAM - CONAMET 2013, Procedia Materials Science 8 (2015) 978 - 985 p

9 ASTM INTERNATIONAL. E 112-13: Standard Test Methods for Determining Average Grain Size. West Conshohocken, 2013, 28p.

10 WAGONER, R.H. Comparison of plane-strain and tensile work hardening in two sheet alloys. Metallurgical Transactions, volume 12A, May, 1981. 877-882 p.

11 INSTITUT DE RECHERCHES SIDÉRURGIQUES, Influence de différents paramètres sur les courbes limites d'emboutissage, Commission des Communautés Européennes, Communauté Européenne du Charbon et de I'Acier, Convention N 6210-77, 1975, 7 - 33 p.

12 XAVIER, M.D.; PLAUT, R.L. Ensaios de tração uniaxiais quase planares aplicados na determinação do parâmetro de estampabilidade CLCo. $49^{\circ}$ Seminário de Laminação - Processos e produtos laminados e revestidos, ABM, Outubro, 2012. $417-427$ p. 